\title{
A Dynamic Non-Energy-Storing Guidance Constraint with Motion Redirection for Robot-Assisted Surgery
}

\author{
Nima Enayati, Eva C. Alves Costa, Giancarlo Ferrigno, Elena De Momi*
}

\begin{abstract}
Haptically enabled hands-on or tele-operated surgical robotic systems provide a unique opportunity to integrate pre- and intra-operative information into physical actions through active constraints (also known as virtual fixtures). In many surgical procedures, including cardiac interventions, where physiological motion complicates tissue manipulation, dynamic active constraints can improve the performance of the intervention in terms of safety and accuracy. The non-energy-storing class of dynamic guidance constraints attempt to assist the clinician in following a reference path, while guaranteeing that the control system will not generate undesired motion due to stored potential energy. An important aspect that has not received much attention from the researchers is that while these methods help increase the performance, they should by no means distract the user systematically. In this paper, a viscosity-based dynamic guidance constraint is introduced that continuously redirects the tool's motion towards the reference path. The proportionality and continuity of generated forces make the method less distracting and subjectively appealing. The performance is validated and compared with two existing non-energy-storing methods through extensive experimentation.
\end{abstract}

\section{INTRODUCTION}

Shared control robot-assisted surgical systems aim at improving and extending human capabilities, by exploiting the advantages of robotic systems while keeping the clinician in charge. One of the most promising features of these systems is the possibility of overlaying pre- and intra-operative information over the operative workspace through different sensory modalities [1]. Constraining the motion of the surgical tool is among the popular approaches that employ haptic capabilities of the operator to intuitively improve the outcome of the surgical procedure in terms of safety, accuracy and cognitive load. Since the introduction of the so called "virtual fixtures" by Rosenberg [2] for a "peg-in-hole" task in a teleoperated environment, interest has been grown in use of software-generated haptic constraints for surgical applications, in both teleoperated [3] and hands-on systems [4]. Active constraints either limit the tool to within a certain space (forbidden region constraints), or attempt to guide the tool in moving along a desired path (guidance constraints).

To implement active constraints, first the geometry of the constraint is generated based on the task data. The majority of the works in the literature assume that the constraint geometry is defined a-priori. Nevertheless, there are methods that try to autonomously build the constraint geometry from medical images [5], [6]. The definition of the active constraint can change during the task. These "dynamic constraints" have

Nima Enayati, Giancarlo Ferrigno, and Elena De Momi are with the department of electronics, information and bioengineering, Politecnico di Milano, 20133, Milan, Italy. \{nima.enayati, giancarlo.ferrigno, elena.demomi \}@polimi.it.

Eva C. Costa is with is with the department of industrial electronics, University of Minho, Braga, Portugal. evalvescosta@gmail.com. particular application in surgical tasks, where the constraints can be designed, for instance, to track organs as they deform or move during the operation. Robot-assisted beating heart surgery is an example of procedures that can benefit from dynamic constraints. Their application in constraining the user's motion has been shown by Ren et al. [7] for the surface of the beating heart and by Navkar et al. [6] for the internal ventricle.

Once the geometry of the constraint is defined and the relative configuration of the tool is known, an enforcement method generates appropriate force/torques accordingly. The focus of this paper is this latter step of active constraint implementation. Although a wide variety of constraint enforcement methods can be found in the literature, the majority of the methods use some form of a linear function of the proximity of the tool and the constraint geometry. A comprehensive review of the methods for generating and enforcing active constraints can be found at [8].

The enforcement of admittance-type constraints is different from that of the impedance-type group. Admittancetype forbidden region constraints can be implemented simply by eliminating any commanded motion into the forbidden region. Here, the tool does not move if the user does not apply a force, since the velocity of the manipulator is proportional to the applied force [9]. However, teleoperation systems are typically designed as impedance-type systems, where an impedance-type haptic device is used as the master (while the slave manipulator can be of either the impedance or admittance-type). For impedance-type devices, the forbidden region constraints are enforced as a "virtual wall" and are typically implemented as simple spring-damper constraints [3]. The forces generated in these constraints are principally proportional to the magnitude of the penetration into the forbidden region. A similar method can be employed for impedance-type guidance constraints, where an elastic link attempts to bring the tool back to the desired path [6]. These constraints can lead to unexpected and undesirable motion of the tool. An elastic link stores potential energy and the constraint will pull the tool toward the desired path, regardless of the direction of the tool's motion, as long as the tool is away from the path. The stored energy can cause unintended abrupt motion of the tool when the user relaxes his/her grasp on the haptic device's end-effector. The situation exacerbates for dynamic constraints, where the changes in geometry of the constraint can lead to frequent energetic activity in the robot, diminishing the surgeon's overall control of the procedure by actively forcing the surgical tool. This can raise concerns for patient safety and clinical certification. To overcome these concerns, a few "non-energy-storing" methods have been proposed that constrain the tool's motion by energy dissipation. 
Kikuuwe et al. [10] introduced a non-energy-storing simulated plasticity method aimed at active constraint enforcement that was based on their previous work on frictional guidance constraints [11]. Simulated plasticity uses an anisotropic proxy model that resists motion in the nonpreferred direction by a large yield (plastic) force. In a guidance constraint implementation of this method, when the tool approaches the reference, a small force is applied, and conversely, when the tool moves away from the reference the applied force is large. However, as long as the tool's distance from the reference is not increased the constraint will not act against the user, thus the tool is allowed to move parallel to the reference. In a work on admittance-type hands-on path following by Bettini et al. [12], the user's motion is projected into a subspace of preferred motion directions and the user is guided towards the reference path even in parallel motion. Inspired by this work, Bowyer et al. [13], proposed a dissipative impedance-type constraint that redirects users motion towards the reference path. The constraint enforcement in their approach is based on the elasto-plastic friction estimation method proposed by Hayward et al. [14] for control applications involving friction and haptic rendering.

Both simulated plasticity and elasto-plastic friction succeed in constraining the user's motion in a dissipative manner. The inclusion of motion redirection in [13], improves the efficacy in guiding the user, as experiments show higher accuracy with respect to the trials with no active constraints. However, an aspect that has not been studied in these works is the "quality" of the guidance forces that are applied to the user's hands. Plastic forces lack the proportionality of elastic (to displacement) or viscous (to velocity) forces, and therefore are less suited to be used for guidance constraints, where the forces applied by the human arm should be dynamically counteracted. While guidance constraints aim at reducing the tracking error, in doing so they should cause the least distraction to the user. During a path following task, where the tool frequently crosses the reference path, plasticity-based methods can produce sudden forces that can considerably disturb the user.

A well-known and intuitive non-energy-storing method for constraint enforcement is viscosity. Due to its proportionality to velocity, a forbidden region viscous active constraint can allow slow violation into the forbidden region. While this is in fact a drawback that motivated the development of plastic methods by [10] and [14], it can be less problematic for guidance constraints. Depending on the velocity ranges, involved, viscous constraint enforcement augmented with redirection can actually be a viable option for active guidance constraints.

In this paper, the feasibility of using viscosity-based guidance constraints is studied by introducing an anisotropic viscous guidance constraint that redirects the tool motion towards the reference path. In previous works, no comparison has been made between the outcome of different non-energystoring methods, and the experiments have studied only the improvement with respect to cases with no guidance constraints. Moreover, in these works, the evaluations have been made solely in terms of accuracy and with no discussion on the characteristics of the applied forces or subjective aspects that are of the utmost importance for eventual implementation of these methods in real clinical practice. In this work, the introduced method is compared with two non- energy-storing methods from the literature through extensive experimentation by 22 users. The efficacy of the active constraints is evaluated both objectively in terms of reducing the tracking error and subjectively considering the "quality" of guidance forces.

\section{METHODS}

In this section, first the plasticity-based non-energy-storing methods are briefly reviewed to highlight a few points that can be of help in contrasting these methods and drawing conclusions. Next, the viscosity-based redirecting guidance constraint is introduced.

In this work, scalars are written normal face lowercase, vectors are written boldface lowercase, and normalized vectors are written boldface lowercase with the hat symbol. The constraints are only applied to the Cartesian position of the tool tip $x \in \mathbb{R}^{3}$.

This paper does not present an in-depth discussion on stability. Although viscosity is intrinsically dissipative and does not generate or store energy, choosing a large viscosity coefficient can cause undesired vibrations depending on properties such as the mechanical damping of the haptic device and its sampling frequency. Here, it is assumed that the viscosity (stiffness for plastic methods) coefficient is appropriately chosen not to violate passivity conditions.

\section{A. Plasticity-based methods}

Haptic devices with impedance causality (flow in/effort out) generate force as a function of displacement. Therefore, simulating an ideal rigid or plastic contact with these devices is not feasible without incorporating an elastic phase. Both the elasto-plastic friction model [14] and the simulated plasticity [10] include an initial elastic deformation that saturates at a certain amount of displacement. This can be easily seen in the simplified coulomb model implementation of the elastoplastic friction by Bowyer et al. [13], where the force $f$ at discrete time-step $k$ is calculated as:

$$
f_{k}=\sigma_{0} z_{k}+\sigma_{1} \dot{z}_{k}+\sigma_{2} \dot{x}_{t, k}
$$

where $z$ is an elastic displacement calculated from:

$$
\begin{gathered}
\mathbf{z}_{k}= \begin{cases}\mathbf{z}_{c} & \mathbf{z}_{c}<z_{\text {max }} \\
z_{\text {max }} \cdot \mathbf{z}_{c} /\left\|\mathbf{z}_{c}\right\| & \text { otherwise }\end{cases} \\
\mathbf{z}_{c}=\mathbf{z}_{k-1}+\Delta \boldsymbol{x}_{t, k} \\
z_{\text {max }}=f_{c} / \sigma_{0}
\end{gathered}
$$

It is important to note that in [13], coefficients $\sigma_{1}$ and $\sigma_{2}$ are set respectively to zero and a small value, making the force mainly linearly dependent on the length of the spring $z . f_{c}$ is the maximum force, which depending on the choice of the stiffness coefficient $\sigma_{0}$ defines the maximum length $z_{\max }$ of the spring.

The impedance-type simulated plasticity of [10] behaves similarly, although the plasticity is achieved in a different formulation including a virtual coupling element. The stiffness $\mathrm{K}$ and maximum force $\mathrm{R}$ define the maximum length of the virtual coupling's elastic element.

In guidance constraint implementation of these methods, when the tool deviates from the pathway, the constraint applies an elastic force. The force augments as the error increases, until the maximum elastic length is reached and the plastic phase is started (force saturation). If the user relaxes his/her 
grip on the tool, the elastic link decompresses and can move the tool up to the maximum elastic length of the constraint (3mm in [13]). Another point that should be noted is that if the constraint force is high enough, it may keep the tool tip in the vicinity of the pathway such that the tracking error rarely exceeds the maximum elastic length. In this case, the constraint's behavior approaches an elastic link that by definition is energy-storing. The maximum elastic length can be decreased to reach the plastic phase with a smaller displacement. However, this can cause frequent bouncing due to rapidly saturating forces when the reference is crossed. This is a limitation of impedance-type plasticity-based constraint enforcement, which renders it less fitting for guidance constraint implementation. A viscosity-based constraint enforcement in contrast can provide a more continuous force profile with smoother boundary transitions.

\section{B. Anisotropic viscosity-based guidance constraint}

The anisotropic viscous force can be attained at time-step $\mathrm{k}$ as follows:

$$
f_{k}=\operatorname{sat}\left(b_{k}\left\|v_{k}\right\|, F\right)
$$

$F$ is the maximum guidance force, $v_{k}$ is the tool tip linear velocity and $\operatorname{sat}(x, X)$ is a saturation function defined as:

$$
\operatorname{sat}(x, X)= \begin{cases}X & \text { if } x>X \\ x & \text { otherwise }\end{cases}
$$

$b_{k}$ is an anisotropic viscosity coefficient based on the normalized vectors of tool tip velocity $\widehat{\boldsymbol{v}}_{k}$ and deviation $\widehat{\boldsymbol{d}}_{k}$ (Fig. 1) at time-step k:

$$
b_{k}=B \sqrt{\left(1-\widehat{\boldsymbol{v}}_{k} \cdot \widehat{\boldsymbol{d}}_{k}\right) / 2}
$$

The term under the radical in Eq. (7) generates the anisotropy in a continuous profile such that the viscosity coefficient is at maximum $B$ for orthogonal deviation (when the dot product of $\widehat{\boldsymbol{v}}_{k}$ and $\widehat{\boldsymbol{d}}_{k}$ is equal to -1 ), and zero for orthogonally approaching the reference (the dot product of $\widehat{\boldsymbol{v}}_{k}$ and $\widehat{\boldsymbol{d}}_{k}$ is equal to 1).

To smoothen the boundary crossing with high velocities the viscosity coefficient $B$ can be downscaled when the deviation is less than a threshold $D_{m}$ :

$$
B^{\prime}= \begin{cases}B \frac{\left\|\boldsymbol{d}_{k}\right\|}{D_{m}} & \left\|\boldsymbol{d}_{k}\right\|<D_{m} \\ B & \text { otherwise }\end{cases}
$$

The boundary-downscaled viscosity coefficient $B^{\prime}$ can be used instead of coefficient $B$ in Eq. (7).

Next, the direction of the guidance force is calculated. The approach is simillar to that of [13]. However, in contrast to

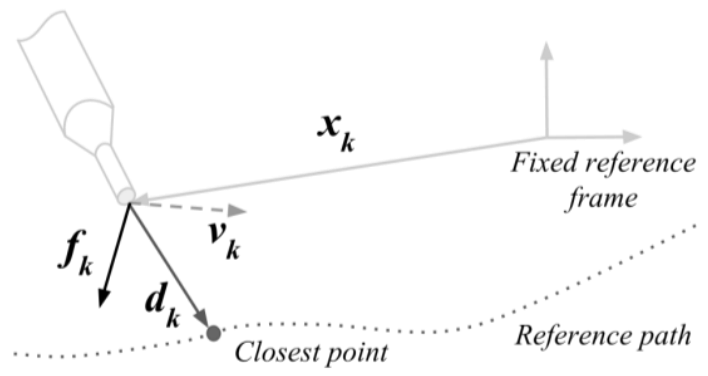

Figure 1. The vectors involved inForce $f_{k}$ attempts to guide the tool tip at $x_{k}$ towards the closest point on the reference

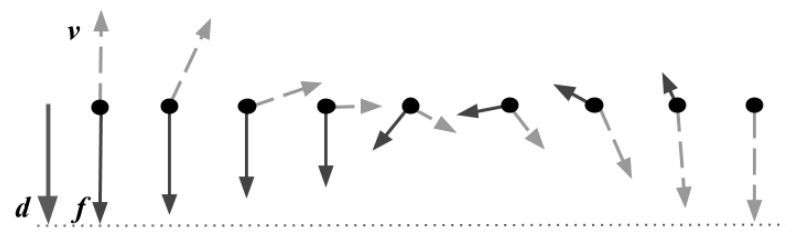

Figure 2. The effect of the anisotropy term on force magnitude and redirection on its orientation for a constant deviation vector $d$ and various normalized velocity vectors. Maximum and zero forces are applied respectively for orthogonal deviation and retraction. A smaller force attempts to guide the tool tip towards the reference in parallel directions of motion.

their fixed redirection angle approach, here the redirection is performed in a continues range of angles, preventing abrupt changes of force direction that can occur in sudden changes of tool direction in parallel motion. The force direction $\widehat{\boldsymbol{f}}_{k}$ is computed as:

$$
\widehat{\boldsymbol{f}}_{k}= \begin{cases}\widehat{\boldsymbol{d}}_{k} & \widehat{\boldsymbol{v}}_{k} \cdot \widehat{\boldsymbol{d}}_{k}<0 \\ \operatorname{rot}\left(\widehat{\boldsymbol{v}}_{k}, \theta_{k}, \boldsymbol{n}_{k}\right) & \text { otherwise }\end{cases}
$$

The rot function rotates the normalised tool tip velocity vector $\widehat{\boldsymbol{v}}_{k}$ around the normal vector $\boldsymbol{n}_{k}$ and through the angle $\theta_{k}$, calculated as:

$$
\begin{gathered}
\theta_{k}=\left(1+\widehat{\boldsymbol{v}}_{k} \cdot \widehat{\boldsymbol{d}}_{k}\right) \pi / 2 \\
\boldsymbol{n}_{k}=\widehat{\boldsymbol{v}}_{k} \times \widehat{\boldsymbol{d}}_{k}
\end{gathered}
$$

In cases where Eq. (11) results in a zero vector, the last non-zero $\boldsymbol{n}_{k}$ can be used.

Finally, the constraint force vector is found from the following scalar multiplication:

$$
\boldsymbol{f}_{k}=f_{k} \cdot \widehat{\boldsymbol{f}}_{k}
$$

The complete course of action of the generated force vector for a normalaized velocity vector is displayed in Fig. 2.

\section{EXPERIMENTS}

To evaluate the described algorithm and to compare its performance with other methods, a haptic virtual environment interface was developed for experimentation. In this section, the implemented systems and the experimental procedure are described.

\section{A. Experimental system}

The experimentation set comprised a virtual environment interface and two Sigma haptic devices (Force Dimension, Switzerland) (Fig. 3). To prevent the computational load of the $3 \mathrm{D}$ graphics from affecting the haptic control loop performance, the visualization and the control loop were executed on separate computers: a desktop computer with 3.2 $\mathrm{GHz}$ Core $\mathrm{i} 7$ processor (Intel Corp.) for the control loop and a laptop equipped with a $2 \mathrm{GHz}$ Core $\mathrm{i} 7$ processor and a 3D display for visualization. The haptic control loop was implemented as an Open RObot COntrol System (OROCOS) application in $\mathrm{C}++$ on a real-time Xenomai-patched Linux kernel. Data such as the tools' pose, forces and task options were sent through UDP communication to the laptop for visualization. The virtual environment was developed using H3DAPI programming interface in $\mathrm{C}++$ and Python. The haptic rate was $1 \mathrm{kHz}$ and the $3 \mathrm{D}$ display rate was approximately $14 \mathrm{~Hz}$. The $\mathrm{C}++$ code of the $\mathrm{AC}$ enforcement methods are available on-line at: 


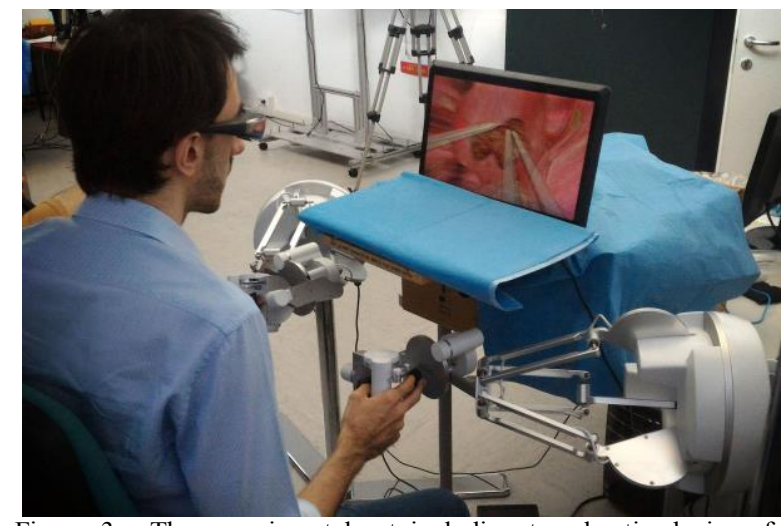

Figure 3. The experimental set including two haptic devices for bimanual operation.

https://github.com/nearmrs/dynamic active_constraints

The parameters used for each method are reported in table I. The maximum guidance force was set to $5 \mathrm{~N}$ for all the three methods. The objective of this work was to prevent the guidance methods to become too aggressive. Otherwise a higher magnitude of force could have been set. The stiffness coefficients were chosen to have a maximum elastic length of $3 \mathrm{~mm}$ for the plastic methods.

\section{B. Experimental procedure}

The experiment consisted of a static task and a dynamic one. To render the static tasks more challenging a tensionand-cut operation was simulated that required the usage of the user's both hands. The dominant hand carried the cutting tool and another tool was used by the non-dominant hand to tension the cutting region. Only the cutting tool was subject to guidance constraint and the tensioning tool displayed solely tissue interaction forces. To perform a cut, the user had to first position the tensioning tool close to the region of interest and press the tissue until a semi-transparent circle appeared. Penetrating the tissue in the circle's inner region with the cutting tool left a color mark on the tissue that_simulated a cut.

\begin{tabular}{cccccc}
\multicolumn{4}{c}{ TABLE I. Parameters Used in Each Method } \\
\hline \multicolumn{2}{c}{ Kikuuwe et al. } & \multicolumn{2}{c}{ Bowyer et al. } & \multicolumn{2}{c}{ Viscosity-based } \\
\hline Param. & Value & Param. & Value & Param. & Value \\
\hline R & $5 \mathrm{~N}$ & $f_{c}$ & $5 \mathrm{~N}$ & $F$ & $5 \mathrm{~N}$ \\
$\mathrm{~F}$ & $0.5 \mathrm{~N}$ & $\sigma_{0}$ & $1667 \mathrm{~N} / \mathrm{m}$ & B & $80 \mathrm{Ns} / \mathrm{m}$ \\
$\mathrm{K}$ & $1667 \mathrm{~N} / \mathrm{m}$ & $\sigma_{1}$ & $0 \mathrm{Ns} / \mathrm{m}$ & $D_{m}$ & $0.002 \mathrm{~m}$ \\
B & $2.5 \mathrm{Ns} / \mathrm{m}$ & $\sigma_{2}$ & $2.5 \mathrm{Ns} / \mathrm{m}$ & & \\
\hline
\end{tabular}

If the penetration exceeded $5 \mathrm{~mm}$, a darker color alteration signaled the user to retreat. These steps are depicted in Fig. 4. The reference path was displayed as a semi-transparent blue line, and a bar on the screen showed the distance of the tool tip from the closest point on the path in millimeters. In the static task the user was asked to tension and cut along the curved contour of a mass (see Fig. 4). In the dynamic task, the simulated environment oscillated parallel to the screen with a rate of $0.3 \mathrm{~Hz}$ to approximate respiratory motion without deformation. Here the user was asked to use only the dominant hand and to follow the assigned contour with the minimum possible deviation. The motion of the user was downscaled by a factor of 3 . To compensate for the limited workspace of the haptic device, the end-effector button was used as a clutch to couple the displacement of the tool with that of the haptic device's end-effector. The following four cases were tested for each task:

- UN - Unconstrained motion

- PL - Simulated plasticity of Kikuuwe et al.

- PLR - Plastic friction with redirection by Bowyer et al.

- VR - Variable viscosity with redirection

The order of the methods was chosen randomly in each trial to prevent any learning bias. Before starting the experiment, each subject read a document that explained the concepts of guidance constraints, implemented methods and the procedure of the experiment. Then the users were given as much time as they needed to train with a square-shaped path. During preliminary tests, it was observed that the subjects were able to benefit decisively better from the guidance constraints when they were told in what fashion the constraint attempted to guide them. For example, a subject can get confused by redirection forces if he/she is not aware of their presence. Therefore, during the training phase the basic ideas of the methods were explained to the subjects while they practiced. The average of training time per user was 23 minutes (minimum of $13 \mathrm{~min}$ and maximum of $47 \mathrm{~min}$ ). The average total time of experiments per user was 58 minutes.

To estimate the subjective performance of each method, the subjects were asked to give a score from zero to ten, describing how distracting each guidance method appeared to him/her. They were told the lower end represents a highly distracting guidance, displaying rapidly changing forces, and the higher end represents no distraction as in unconstrained motion. The users were informed in advance that after the

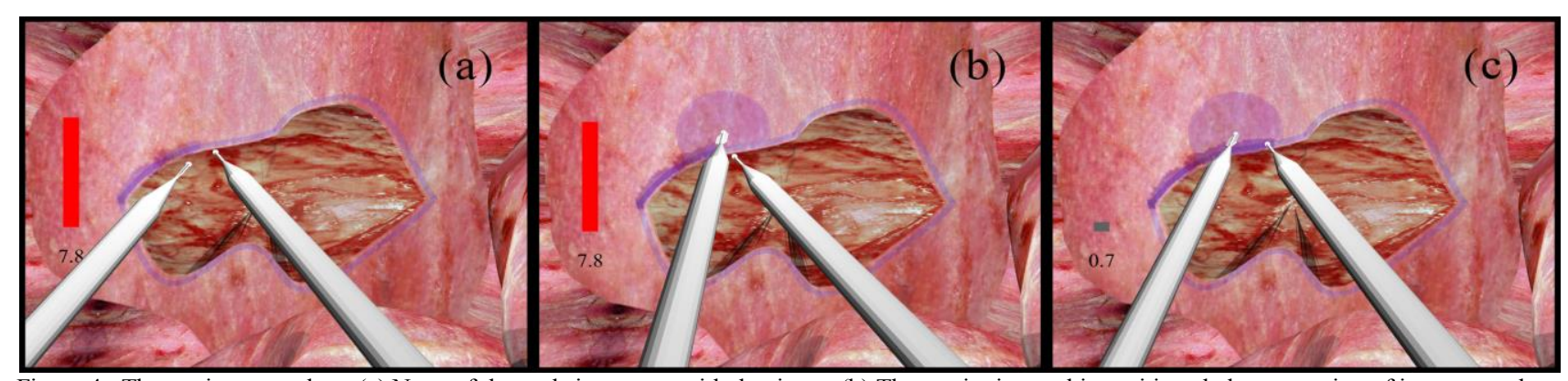

Figure 4. The cutting procedure. (a) None of the tools in contact with the tissue. (b) The tensioning tool is positioned close to region of interest on the tissue. (c) The cutting tool performs the cut inside the tensioning circle. 

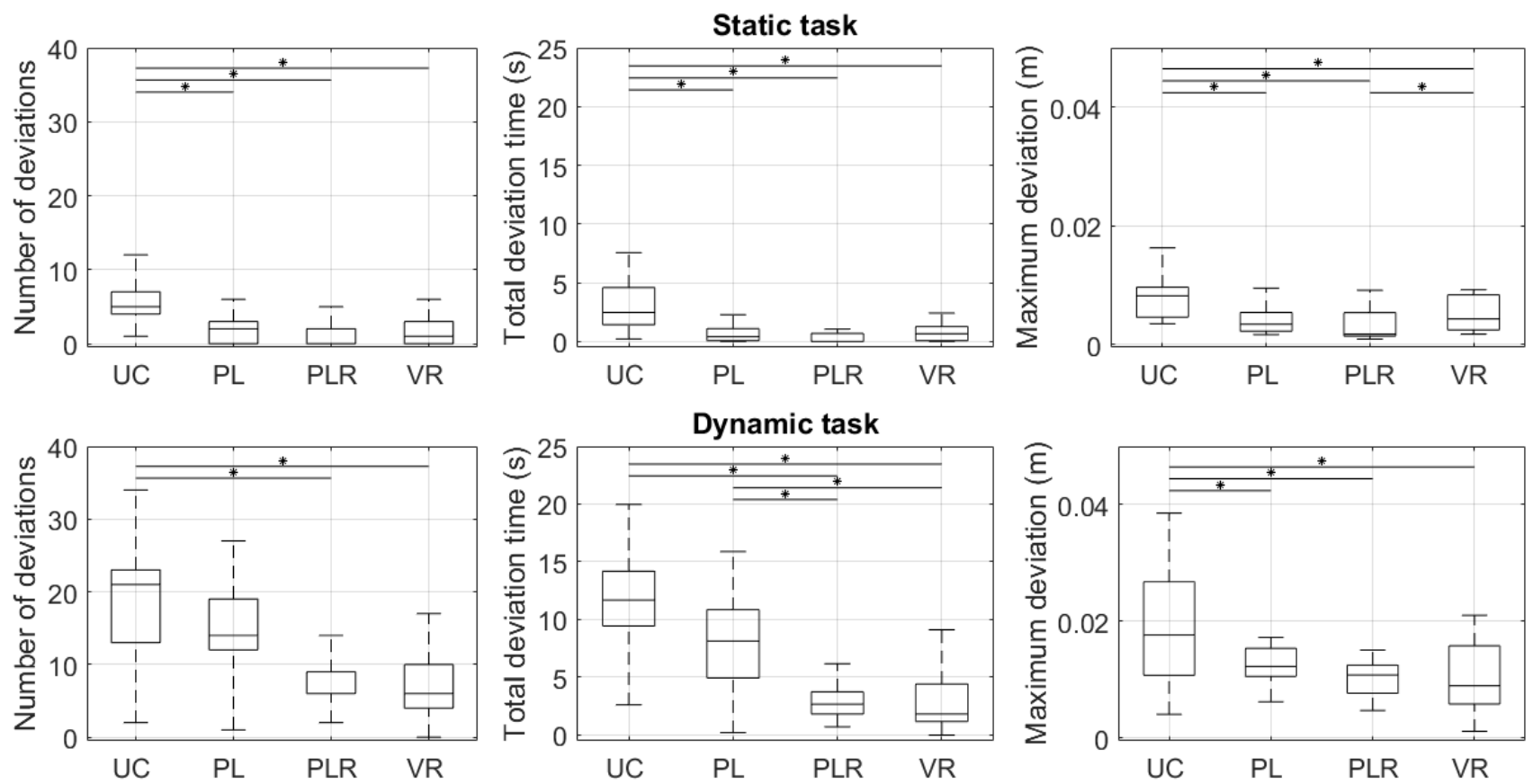

Figure 5. Accuracy enhancement results for both tasks. Plots from left to right: number of deviations larger than $1 \mathrm{~mm}$, the sum of the duration of these deviations and the maximum deviation. Methods are: UC (UnConstrained), PL (Plastic), PLR (PLastic with motion Redirection) and VR (Viscose with motion redirection).

completion of each task (except for the unconstrained cases) they will be asked for the score. 22 subjects (11 females, 11 males), ranging from 18 to 30 years old, participated in the experiment, none of whom had considerable experience with haptic systems or surgical interventions.

\section{RESULTS AND DISCUSSION}

The path following deviation was calculated only for the cutting tool and only when it was in contact with the tissue. For each user, 8 acquisitions were recorded ( 2 tasks and 4 methods). To estimate the objective performance of the methods in terms of accuracy, the number of deviations larger than a threshold of $1 \mathrm{~mm}$ and the total duration of these deviations were calculated for each subject and task. Naturally, the deviation threshold is a task specific parameter and has to be chosen according to the performance requirement of the surgical procedure. Since the analysis here is not devoted to a specific surgical task, the threshold was set relative to the dimensions of the reference path. In addition, the maximum deviation from the reference path was found for each acquisition. The distributions of number of deviations above the threshold, total deviation duration and maximum deviation for each acquisition are visualized in Fig. 5 and their median values are reported in table II. None of the sets were normally distributed $(\mathrm{p}<0.005)$ and therefore, the nonparametric pairwise Friedman test $(p<0.05)$ was performed to determine the statistical significance of the sets.

The results suggest that all the three guidance constraints have enhanced the accuracy in the static task compared to the unconstrained cases. The enhancements are more considerable in the dynamic case since the task was more demanding. Considering the number of deviations larger than
$1 \mathrm{~mm}$ and the total duration time, the PL method did not demonstrate statistically significant improvements compared to the unconstrained case, while it reduced the maximum deviation. The methods with motion redirection (PLR and VR) demonstrated a significant improvement all the three accuracy metrics. Furthermore, these methods have reduced the variability of the performance among the users compared to the unconstrained sets (and compared to the PL method in deviation count and time). The variability of the deviations is a direct result of the disparate user skills. During the experiments, it was observed that some subjects had difficulty in performing the tasks efficiently due to their lack of experience with bimanual operation in a virtual environment through haptic interfaces. Since the guidance forces had a relatively small magnitude, the subjects could easily act against them in moments of confusion. The subjects who showed no/little struggle in using the setup, were able to benefit more from the methods with redirection by relaxing their grip and allowing the active constraint to direct them towards the reference path., PLR reduced the variability relatively more, that can be due to its more restrictive forces.

Although PLR and VR methods demonstrated comparable performances in accuracy enhancement, the levels of distraction were not alike. The averages of 44 scores (22 users and 2 tasks) given by the subjects on the distraction level of each method are reported in table III. The PLR method received a considerably lower score from the users, suggesting that the subjects found the method more disturbing. These results can confirm the discussions made earlier in this paper on the disadvantages of using plastic redirectional methods for guidance constraints due to rapidly saturating forces. This behavior can be seen in Fig. 6, where 
TABLE II. Median values of maximum deviation distributions

\begin{tabular}{lcccc}
\multicolumn{5}{c}{ showed in Fig. 5 $(\mathrm{m})$} \\
\hline Method & UC & PL & PLR & VR \\
\hline Static Task & 0.008 & 0.004 & 0.002 & 0.004 \\
Dynamic Task & 0.018 & 0.012 & 0.011 & 0.009 \\
\hline
\end{tabular}

TABLE III. Average of scores given by users

\begin{tabular}{|c|c|c|c|c|}
\hline Method & & $\mathrm{PL}$ & PLR & VR \\
\hline Average score & & 7.6 & 5.3 & 7.5 \\
\hline \multicolumn{5}{|c|}{ TABLE IV. Average time (s) } \\
\hline Method & $\mathrm{UC}$ & $\mathrm{PL}$ & PLR & VR \\
\hline Static task & 98.9 & 90.1 & 80.2 & 86.0 \\
\hline Dynamic task & 33.4 & 27.0 & 22.7 & 22.3 \\
\hline
\end{tabular}

the guidance forces generated by different methods recorded in a static task are depicted. Quick saturation of the forces in the PLR method causes the tool to bounce frequently around the reference path. The VR method on the other hand shows a less oscillating and a more proportional behavior as expected. The average of the force magnitude for all acquisitions was calculated as $1.4 \mathrm{~N}, 2.7 \mathrm{~N}$ and $1.7 \mathrm{~N}$ respectively for PL, PLR and VR methods. Low average force of the PL method is due to the fact that it does not oppose parallel deviation, and thus it applies force less frequently. The average task time presented in table IV, shows that users have finished the tasks faster when methods with motion redirection (PLR and VR) were used, that can implicitly imply ease of use and reduction of cognitive load.

The overall results show that the introduced viscosity-based constraint can achieve accuracy enhancements comparable to those of more restrictive plastic constraints, yet exhibit better subjective performances.

\section{CONCLUSION}

In this paper, a dynamic non-energy-storing guidance constraint was introduced. In contrast to conventional elastic methods, non-energy-storing constraints do not create undesired motions and only dissipate the user's energy. A viscosity-based guidance active constraint was introduced that does not limit the freedom of the human user and attempts to guide the motion towards a desired path in a least aggressive way. Experimentation confirmed the feasibility of
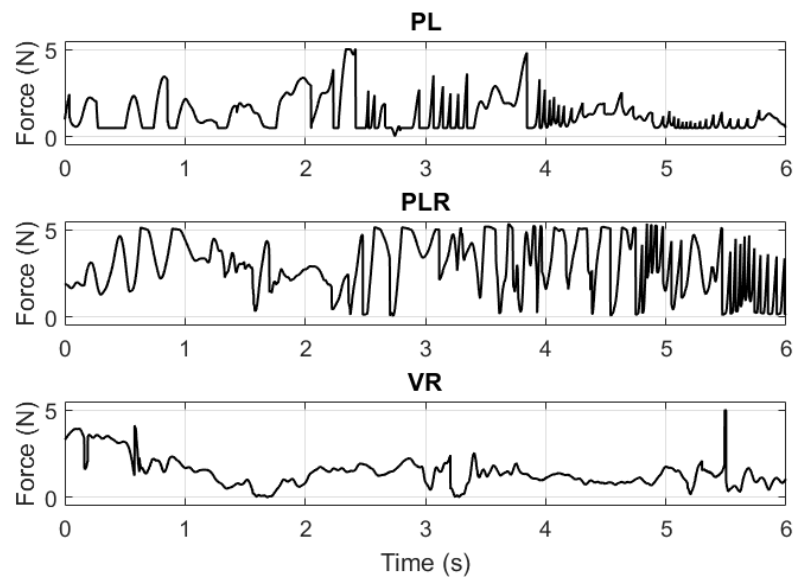

Figure 6 . The magnitude of the guidance force generated by each active constraint methods in a section of a static task. implementing viscosity-based guidance constraints, augmented with motion redirection for dynamic tasks. The introduced method can improve the accuracy of dynamic path following while distract the user less compared to other stateof-the-art methods.

This work argued that although guidance active constraints are designed to enhance accuracy, subjective aspects and the quality of generated forces must be carefully examined too. Future works should introduce more descriptive and quantitative metrics for the evaluation of guidance forces. Moreover, the presence of human in the control loop makes the evaluation of active constraints challenging. The performance could greatly vary depending on the skills of each subject. In future, these methods must be tested by expert users in tasks similar to those of surgical procedures. As commercial surgical robots with haptic capabilities are soon to appear, it can only be expected to see more research efforts expended in this field.

\section{REFERENCES}

[1] N. Enayati, E. De Momi, and G. Ferrigno, "Haptics in Robot-Assisted Surgery: Challenges and Benefits," IEEE Rev. Biomed. Eng., 2016.

[2] L. B. Rosenberg, "Virtual fixtures: Perceptual tools for telerobotic manipulation," Proc. IEEE Virtual Real. Annu. Int. Symp., pp. 76-82, 1993.

[3] J. J. Abbott and a. M. Okamura, "Virtual fixture architectures for telemanipulation," 2003 IEEE Int. Conf. Robot. Autom. (Cat. No.03CH37422), pp. 2798-2805, 2003.

[4] M. Jakopec, S. J. Harris, F. Rodriguez y Baena, P. Gomes, and B. L. Davies, "The Acrobot ${ }^{\circledR}$ system for total knee replacement," Ind. Robot An Int. J., vol. 30, no. 1, pp. 61-66, Feb. 2003.

[5] J. W. Park, J. Choi, Y. Park, and K. Sun, "Haptic Virtual Fixture for Robotic Cardiac Catheter Navigation," Artif. Organs, vol. 35, no. 11, pp. 1127-1131, 2011.

[6] N. V. Navkar, Z. Deng, D. J. Shah, K. E. Bekris, and N. V. Tsekos, "Visual and force-feedback guidance for robot-assisted interventions in the beating heart with real-time MRI," 2012 IEEE Int. Conf. Robot. Autom., pp. 689-694, May 2012.

[7] J. Ren and R. Patel, "Dynamic 3-D virtual fixtures for minimally invasive beating heart procedures," Med. Imaging, ..., vol. 27, no. 8, pp. 1061-1070, 2008

[8] S. Bowyer, B. Davies, and F. R. y Baena, "Active constraints/virtual fixtures: A survey," IEEE Trans. Robot., vol. 30, no. 1, pp. 138-157, 2014.

[9] J. Abbott, P. Marayong, and A. Okamura, "Haptic virtual fixtures for robot-assisted manipulation," Robot. Res., pp. 49-64, 2007.

[10] R. Kikuuwe, N. Takesue, and H. Fujimoto, "A control framework to generate nonenergy-storing virtual fixtures: Use of simulated plasticity," IEEE Trans. Robot., vol. 24, no. 4, pp. 781-793, 2008.

[11] N. Takesue, R. Kikuuwe, A. Sano, H. Mochiyama, and H. Fujimoto, "Tracking assist system using virtual friction field," 2005 IEEE/RSJ Int. Conf. Intell. Robot. Syst., pp. 3927-3932, 2005.

[12] A. Bettini, P. Marayong, S. Lang, A. M. Okamura, and G. D. Hager, "Vision-assisted control for manipulation using virtual fixtures," IEEE Trans. Robot., vol. 20, no. 6, pp. 953-966, 2004.

[13] S. a Bowyer and F. Rodriguez Y Baena, "Dynamic Frictional Constraints in Translation and Rotation," 2014 IEEE Int. Conf. Robot. Autom., pp. 2685-2692, 2014.

[14] V. Hayward, B. S. R. Armstrong, F. Altpeter, and P. E. Dupont, "Discrete-time elasto-plastic friction estimation," IEEE Trans. Control Syst. Technol., vol. 17, no. 3, pp. 688-696, 2009. 